\title{
Studying cue-dependent and intentional forgetting in organizations: a methodological approach
}

\author{
Jennifer Haase \\ Potsdam University \\ Jennifer.Haase@wi.uni-potsdam.de
}

\author{
Arnulf Schüffler \\ Ruhr-University Bochum \\ arnulf.schueffler@ruhr-uni-bochum.de
}

\begin{abstract}
The establishment of new working routines as an adaptation to changing working conditions requires not just the learning of the new content but also the forgetting of the old knowledge. To further understand exactly how old working routines are put behind, four experiments within a realistic working environment are designed. All experiments are based on group work of three participants, thus individual learning and forgetting can be measured, as well as the group performance all together. The experimental design and the implementation of measuring forgetting is explained in detail.
\end{abstract}

\section{Adaptation to changing working conditions}

The increasing digitalization and thus reformation of work environments lead to an enfolded demand of adaptive working procedures. When the environment changes, the behavior has to adapt to be effective. This modification includes the process of learning as well as forgetting [1]. But whenever change in organizations is discussed, the topic of learning is often leading whereas the precise focus on how to forget knowledge or well established working routines is less well studied [2]. This paper aims to describe precisely how an experimental design can be used to measure a less manifest concept like forgetting and apply it to the context of industrial working routines. Before diving into the design of the experiments, the basic concepts like knowledge, learning and forgetting are explained.

\subsection{Knowledge in organizations}

Organizational knowledge describes all the data and information, the knowledge and skills an organization and accordingly its members contain in order to fulfill its mani- fold tasks on every day basis [3]. Thus knowledge is the key asset for the organizations success [4]. It is assumed, that organizations have a memory and acquire knowledge on the same premises like individuals [5]. Whereas individuals are more flexible in adjusting personal knowledge through learning and forgetting, an organization has less precise mechanisms to manage its knowledge. Because organizations learn and forget through their members [6], coordination processes are needed to be added to the employees' cognitive capacities in learning and forgetting [7], [8]. Thus learning and forgetting on the organizational level entail more than just the sum of its employees individual learning and forgetting [2]. For example, Akgün [9] could show that groups could easier forget knowledge and so far established procedures when outsiders were included into the team and performed together. Thus forgetting in organizations is constrained to the interplay between individual and group performances.

One way to operationalize such coordination and establish quality and reliability in working processes is through routines [10]. In essence, routines are repeating, contain observable patterns and actions which are distinct from another, with several individuals being involved [11]. Routines can be formal or informal. The first are implemented through decision of the organization and written down in form of process mapping. The latter are established through practice and shared via observation and socialization [12]. This interdependence of coworkers learning from each other and influencing each others behavior points to the fact that, again, organizational performance through routines is not just the sum of individuals' performance. It also includes team or group dependent effects.

\subsection{Forms of forgetting}

The ability to forget can be seen as the natural pendent to learning, as a fundamental necessity to effectively cope with an ever changing world [13]. Information can be for- 
gotten through deleting, overwriting, suppression or selection [14]. In detail, in the literature several ways of forgetting are distinguished: forgetting via decay, due to interferences and cue-dependent forgetting. Decay describes forgetting by simply missing the repetition of an information. Since memory fades with time (principle of „use it or lose it"), the memory once formed in the brain disintegrates when not used [15]. Interference as a form of forgetting happens when new information gets confused or combined with other memory content during the encoding process [16].

Cue-dependent forgetting describes that the recall of a memory item, formerly activated via encoding an associated cue, does not take place any more. A cue in this context, is a stimulus of any sort, but in some way sensible, which is associated via a specific trace that brings the memory to consciousness or into behavior. In terms of forgetting the association between cue and memory can be as much weekend that a recall does not become activated any more [14] or the cue itself becomes substituted, looses its uniqueness due to an overload [17], [18] or is absent. A missing cue leads to a not-activation of the associations between cue and memory, so the recall of this certain memory does not take place [19].

Another perspective on forgetting is intentional forgetting, which describes the active and regulated suppression of information when the memory is unwanted [20]. This form is an active and voluntary facet of forgetting, whereas forgetting in the forms mentioned before is mostly understood as happening accidentally [21]. Thus whenever memory content is voluntarily inhibited, we speak of intentional forgetting.

Intentional forgetting could be interpreted as synonymous to unlearning. Since the term finds itself in an ongoing debate about its correctness or even existence (see e.g. [22]), we chose to distance our work from it. Instead, we use the concept of intentional forgetting to describe the phenomenon that people can actively manipulate recall in order to adapt to changing working conditions. Whether those memories could be still activated, or are "really" forgotten due to decay is unknown, and actually not of importance (compare [23]). The behavioral outcome of notusage of memory is what we focus on.

\subsection{Research focus}

As Klammer and Gueldenberg [24] stated in their review about organizational forgetting, there is much need for further investigation into the dynamics of learning and forget- ting. Since much is already known about how knowledge is acquired through learning (e.g. [16]), the focus here lies on how individual forgetting can aid the organizational knowledge management process. More so, since intentional forgetting is perceived as a major driver for organizational success and innovative motor [25]. From the perspective of an organization which needs to manipulate forgetting in order to adapt to changing environmental conditions, we take the question of how to do so. Because cue-dependent forgetting as well as intentional forgetting are forms that can be influenced from the outside, a company could use those to manage its applied knowledge. In case outdated information or even routines need to be put behind, we assume that the environment can be manipulated in such a way, that employees are eased with the forgetting process. For example, via managing the cue perceivableness and thus information accessibility which would have a direct impact on the memory which is retrieved and hence can be used [21]. Classic experiments addressing forgetting usually analyzed word-list recognition (e.g. [26], [27], [28]) which does not entail much information about the mechanism on learning and forgetting in complex knowledge-intensive working routines. We constructed an experimental setting which allows to test for diverse influences on cue-dependent forgetting as well as intentional forgetting under laboratory working conditions but still within a realistic work environment. The basic goal is to deepen the understanding of how retrieval cues can be varied, in order to enhance the forgetting of work related activities and routines, both on the group-level as well as for individuals. The effectiveness of intentional forgetting is tested using an experimental setting which implicitly requests the participants to not-use old information and instead apply newly learned information throughout all experimental conditions (see 3.3 Four different experimental conditions).

\section{Applying a hybrid simulator envi- ronment}

The experiments take place at the „Forschungs- und Anwendungszentrum 4.0" (FAZI, research and application centre industry 4.0) at Potsdam University, Germany, which is a training factory where real production processes are replicated for research and demonstrative purpose [29]. The FAZI is a hybrid simulator environment combining the advantages of a digital factory with those of a model factory. Thus the simulation does follow a physical as well as computer based approach. Developed following the principles of 
mutability [30], the main advantage is the flexibility with which any industrial production can be mimicked. Physical models are used to represent relevant production objects like machines, robots and products. Using a combination of software and hardware, production specific elements can be simulated most appropriately using either a physical or digital approach or a combination of both. Thus the environment presents the base which can be used for various industrial production contexts and its elements can be individually programmed to fit the production demands. For example, machines in our scenario are realized using physical computer blocks attached to the production line plus software to apply the user interface for the machine as well as to present optical and audible feedback specific for the machines [31], [32].

Using both physical as well as digital elements, a complex scenario is created which allows participants to fully immerse into the scenario (see Figure 1). Even more so in addition with a cover story about the company and its need for getting its production process tested. Participants interact during the experiments with the machines and robots, as well as with the products on the production line. They also move around the room in order to get material for their process. Overall, this immersion which is created enhances learning through concrete and specific actions and operations [33].

For our experiments, the production of knee joint implants is simulated, based on the real production process of an associated company. In order to adjust the original production process to the laboratory conditions, several steps were made: first the original process was assessed step by step and then prioritized to the basic tasks. Next, those were logically combined, so overall three workers are needed for the whole process (the number of three was chosen, since three workers are already a team, but the number is feasible to plan experiments with). Then the tasks within those three positions were aligned so all have nearly the same amount of tasks (W1 $n=47$, W2 $n=47$, W3 $n=49$ ) with approximately same difficulty and usage of technique and material. Further, all three positions are planned to have a nearly similar amount of tasks which change at the goalprocess (W1 $n=21$, W2 $n=18$, W3 $n=19$ ). Least, tasks were adjusted so the performance of the workers can be measured at all times using paper-work, machine-interaction or operations we can see via eye-tracker.

Real photo-material and sounds from the original production process are used in order to create best-possible realistic production conditions. Further, presenting photos on digital screens of the work pieces the development of the blank throughout the whole process is demonstrated. Thus it is possible for the workers to get a feedback about their performance, since a wrong setting or handling of the machines would result in a distorted form of the work piece represented using different pictures of the real knee joint implant.

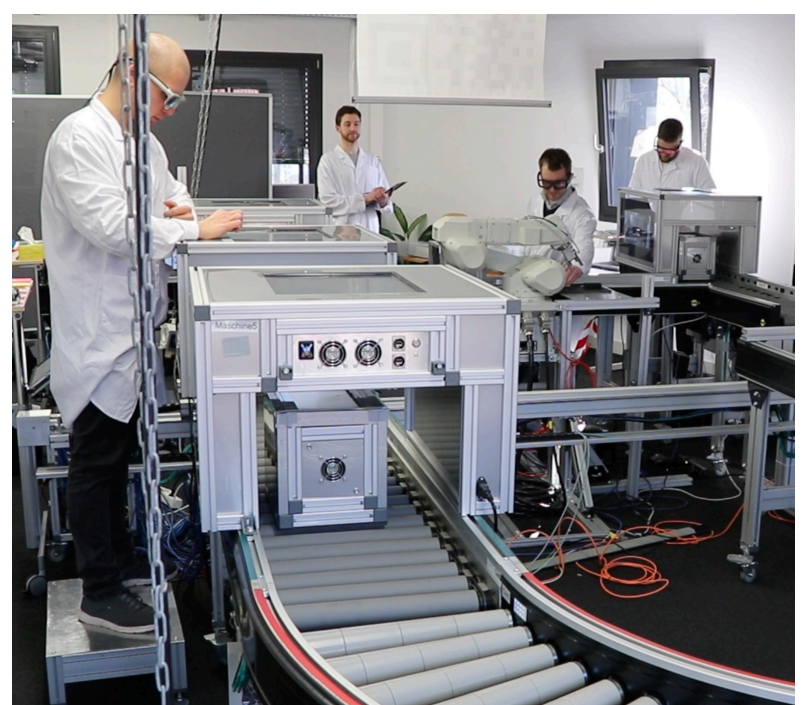

Figure 1. The experimental setting at the laboratory with participants and test leader.

\section{Experimental design}

The basic idea behind the experiments are to first led participants learn a production process (which would most probably not be known by the participants) and then let the working routine be over-learned through repeated practice in order to establish a fixed mental routine about the learned material. In a next step, parts of this routine are made obsolete, so intentional forgetting is needed in order to adapt to the new working conditions. Cue-dependent forgetting is operationalized through different experimental conditions. Overall, we tried to mimic realistic change-conditions within a company as close as possible.

\subsection{The experimental production process}

The simulation of the production process includes three working positions: worker one mills and grinds the blank, worker two uses a laser and polishes the item, and worker three is responsible for sterilization and packaging of the final product (see Figure 2). All work- 
ers use the PDA-Terminal for the registration of their work piece in the same way and in the warehouse the work pieces are prepared by the test leader for worker 1 to be used again in the production process.

The participants are introduced to the experiment via a video with an experimenter illustrating the scenario of the knee implant manufactory. All participants wear laboratory coats to enhance the feeling of a production setting. During the first session each worker is learning the production process using a written manual with step-by-step instructions. They then work on three items in series. The workers are in a production chain and depend on each others performance. After the third product is finished, the worker continue their work but without the manuals as a guidance (for exceptions see 3.3.2 Experiment 2). Using the example of worker one, s/he would learn to register the products into the PDATerminal based on the production plan and then find the corresponding physical blank in the storage. S/he then learns to check whether the blank fits the requirements and prepares the machine for the milling. After that is done correctly by operating on the user interface, the item is ground and transferred on the production line to the next worker. The process includes the usage of the interface of the machine, a computer, a hand-held scanner, a slide gauge, diverse post-its and papers, along with a pencil and highlighters.

Interactions between the workers are encouraged whenever something unusual happens, when items entail mistakes or the working process holds up. Since the whole team is measured on their joined performance, they are motivated to work together to produce as many failure-free knee implants as possible.

\subsection{The experimental procedure}

All experiments include three sessions: 1. the first laboratory session where participants learn their part in the production process, 2. for the next two weeks they practice their working routine twice at home for around 20 minutes each using an online application, 3. second laboratory session where they learn a similar but somewhat changed process of the production line (see Figure 3.).

The procedure of the experiment is the same for all four experimental conditions: potential participants register at an onlineplatform and chose a date for the first session. A team of three participants then start the session with filling out a questionnaire about general self-efficacy [34] and a questionnaire measuring prior knowledge about production processes. Than they watch a short instruction video to get into the whole factory scenario (called focus-process). They then get eye-tracking glasses, for us to follow their gaze during the experiment (and thus their performance can be coded afterwards). The learning and performing of the production process follows for around one hour. Afterwards participants fill out measures for presence [35], [36], specialized self efficacy and awareness of control [37] and sociodemographic data. These concepts are expected to have a significant impact on the participants performance and are needed to control for influencing factors.

A week after the first session, they receive an Email with a link and instruction for an online application, mimicking the production process for the workers, respectively. This is done to make sure the explicit knowledge of the production process is learned and absorbed so deeply, that a mental image of the formal routine establishes. They can train as long as they want, but we recommend about 20 minutes, one and two weeks after the first session, respectively. At the third week they come back to the laboratory and basically repeat the whole process. Right from the start they are informed that some changes were made to the production process, which is done in a way that it should interfere with the so far established knowledge. The changed process (called goal-process) is framed as a result of the fusion with an international company, so especially quality standards are adapted to fit international norms. Special-

Figure 2. Production line assembly at the FAZI. 


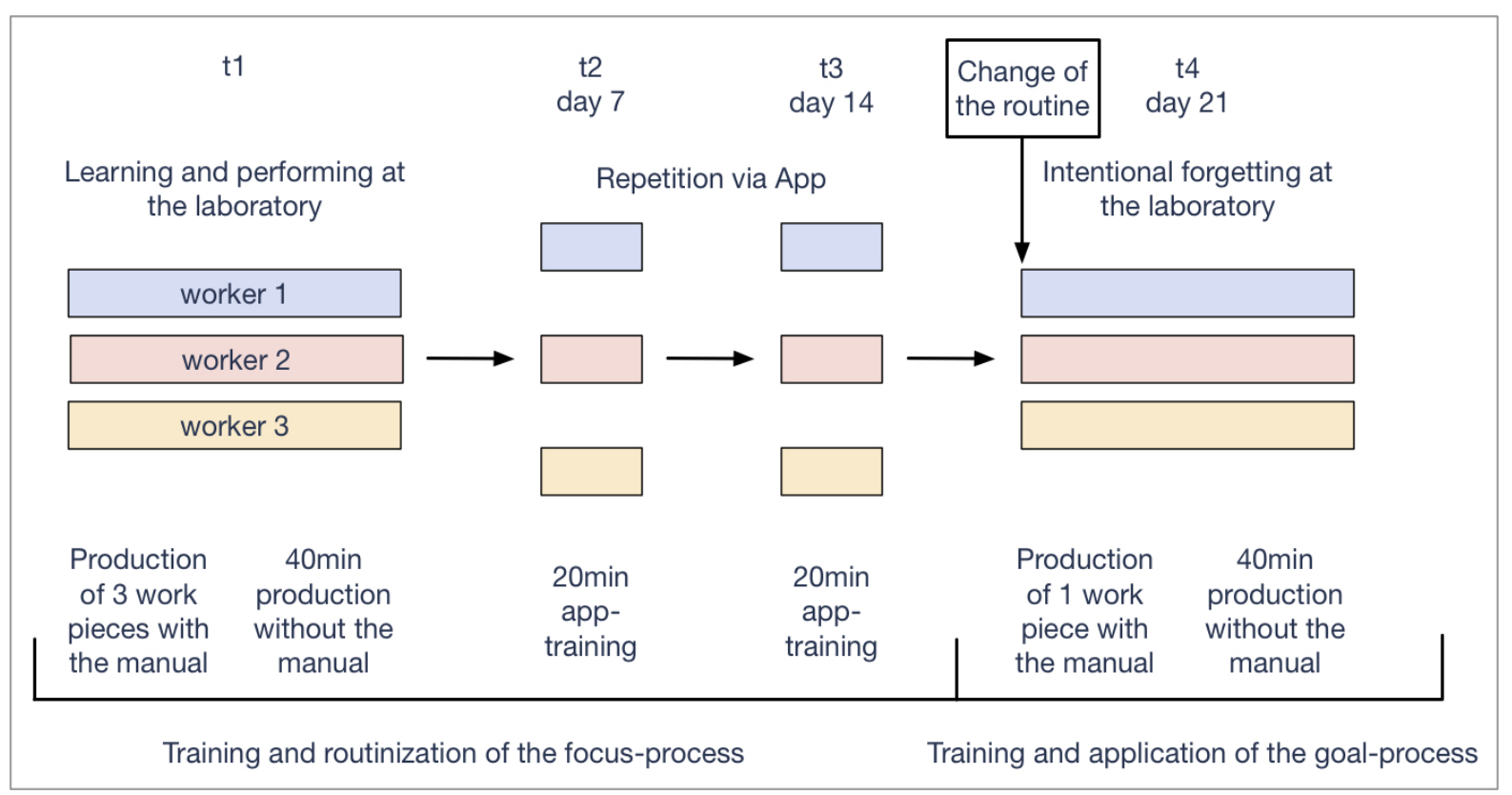

Figure 3. Schema of the experimental procedure.

ized self efficacy and awareness of control [37] is measured repeatedly and the workers use again a manual to learn the changed process and get one test trial before producing as many correct products as possible according to the new production plan. After working for around one hour, they answer a more comprehensive block of questionnaires and tests, assessing presence [35],[36], general memory capacity [38], Fluency [39] and subjective switching costs relating to the first vs. the second laboratory session.

\subsection{Four different experimental conditions}

Although all experiments follow the basic schema described above, some elements are varied during four experimental conditions. The first two directly address cue-dependent forgetting, whereas during the following two experiments, common factors of influence on the forgetting process are analyzed: time restriction as well as reward and punishment.

3.3.1. Experiment 1. Organizational forgetting is a combination of forgetting on individual, group- and organizational level. The experimental design is based on group work, with workers' performance depending on the others. Although it is assumed that the forgetting process on the group level depends mainly on the individual forgetting, work related activities are more difficult to forget when other workers are like cues by itself to remind about the process. Plus, the knowledge about the informal routines from the focus-process is minimized for all workers when one member enters the group at the goal-process who is already trained in executing this for the others new routine [9]. This is tested within the first experiment, where the design includes a substitution of one worker at the second laboratory session for half of the experimental groups. Thus, it can be derived whether forgetting is aided on the individual and group level, when one group member changes. In theory, forgetting should be quicker and more frequent for the member-substituted groups, because here the cues for the original process are reduced and the knowledge about how processes are coordinated is partly lost [40].

3.3.2. Experiment 2. We assume that the learning material in itself is a strong cue for the memory of the performance [41]. Thus the presentation of those cues should facilitate the remembrance of the working processes. In practice, often times changed routines are established using old material, which can make it hard to forget the old behavioral patterns [42]. The goal for experiment 2 is to test the influence of the presentation of outdates vs. newly adapted information. Based on the standard learning material for all experimental conditions, a short version - like a crib sheet - was composed for the three workers, respectively. Having two forms of crib sheets (one for the focus, anther for the goal-process) all experimental groups $(n=88)$ are split into four subgroups, varying between presentation of no, the correct, the outdated or both crib sheets: all groups see the correct sheet during the first session, one group gets no sheet during the second session, neither 
the right nor the outdated one, one group sees further the sheet for the changes at the second session (so at both times a correct one), another group gets both sheets at the second sessions (so a correct and a wrong one during the second session) and the fourth group sees the sheet from the first session in both sessions (so a wrong one during the second session). This scheme makes it possible to test how the no-cues, the wrong-cues, both cues and the correct-cues-conditions differ in their performances. It is assumed that the group with the correct sheets should outperform the other groups by far.

3.3.3. Experiment 3. The adaptation and learning of a new working process takes more time, than if the original process would have been performed. Still, in practice, this adaptation to the individual learning curve is often enough not planned for, which can lead to slower and oftentimes false adaptation to the new routine [11]. This experiment tries to test the impact of time restriction on the individual and group level. The design is according to the first experiment, also with two groups being compared, one with fixed time target, the other with room for individual dynamic timing. Time restriction is said to be counterproductive for intentional forgetting [43]. Under time restriction it is easier to manage this restriction by using the old trained routine which allows a quicker production than a new routine [11]. It is assumed that the forgetting of the old process is promoted within the individual dynamic timing condition.

3.3.4. Experiment 4. During this experiment, the impact of reward and punishment is tested. The design is similar to experiment 2, with a $2 \times 2$ matrix for punishment of wrongly performing the old process (yes/no) and reward for correctly performing the new process (yes/no). There are inconclusive results in the literature, with examples showing improved forgetting due to punishment [44] and a failed suppression effect due to punishment [11]. The bottom line might be the kind of reward and punishment applied. When the punishment comes in the form of error messages, this might be frustrating for the participant and thus hinder correct learning. Instead, if the reward and punishment comes in financial form, this might increase motivation and thus concentration. Based on classical conditioning theory intentional forgetting should be supported best in case not forgetting of the old routine will be punished and the use oft he new routine will be rewarded [44].

\subsection{Sampling and data acquisition}

The main source for participants will be students from the University Potsdam, Germany, especially from Business studies and Business Informatics. In addition, advertisements for the public is put out on social media und via leaflets to address non-students. The goal is to get a mixture of participants with variation in age and background, with and without practice in production settings. The requirements for the participation are simply the commitment to the strict timeframe and fluent German language skills.

Concerning the sample size, we aim for 22 groups per experimental condition. Thus we assess 44 groups for the first and third experiment, and 88 groups for the second and fourth. The group size results of the calculation of an expected middle effect size of $F=$ 0.30 with an $a=0.05$ and a test power of 0.95 for an Anova (GPower, http://www.gpower.hhu.de). This all together generates a need for 264 groups, á 3 participants, makes 792 participants.

The data we assess during the experiments come from four basic resources: log-data from the machines, the PDA-terminal and the app; paper documents filled out by the workers along with the production process; eyetracker data about the movements and manual tasks from each workers and assessment of questionnaires and tests. Overall, this results in an extensive amount of data. This variety of assessment-forms is necessary in order to increase the data on performance indicators in the production process.

\section{Making forgetting measurable}

Since it is rather complicated to measure memory that is gone, we deduce forgetting indirectly from the performance during the production process. The change of routine at the second laboratory session follows a certain logic: there are tasks which are the same over the two sessions for all three workers (e.g. searching for the correct working plan in the PDA-Terminal), the same for one worker (like preparing the machine for milling for worker one); changing over the sessions for the workers in the same way (like the filing of the paper working plans) or changing for each worker respectively (like measuring the size of the blank in cm vs. in inch for worker one). During the focus-process the general learning capacity is measured by checking the performance of each worker after the first three trials. That works as the baseline of what has been learned about the production process. The training sessions with the app assure the consolidation of the learned material. At the 
second laboratory session the forgetting is then derived from the performance during production time after the first learning trial. Since both, the focus- as well as the goalprocess, entail a precise sequence of actions for each worker respectively, the correct and wrong actions during the experiments can be derived.

Concerning intentional forgetting, this can be assessed focusing on the individual performance in all groups of all experiments. We look at those tasks, which change from the focus- to the goal-process. Whenever a participant first correctly performed an action in the focus-process and then correctly performed the changed element in the goalprocess, intentional forgetting is derived. Because it is assumed that the former correct way from the focus-process is still known but is not applied since the goal-process requires an adaptation of behavior. However, whenever such a changed action is correctly shown during the goal-process, but has not been correctly performed during the focus-process, no intentional forgetting can be assumed, because it is not clear whether the knowledge about the focus-process was encoded at all.

Concerning the cue-dependent forgetting, almost everything associated with the learned material could work as a cue [6]. Thus it can be difficult to filter out the for the individuals working in the production process relevant ones. We concentrated on those which appear most applicable and useful in practice [45], which are team-member substitution (Experiment 1) and the presentation of memorysupporting material for both processes (Experiment 2). Here, we compare the experimental conditions with each other. For experiment 1 , the overall performance of the group and its members of the stable-team-condition is compared with the overall performance of the team members with member-substitution. Thus it can be assessed whether a change of work-group-compilation helps with the intentional forgetting of the old process. Although intentional forgetting is assessed as explained above, the comparison of experimental condition allows conclusions on the work mechanisms of cues. Similar, the performance during the four experimental conditions of the second experiment are compared with each other. Assessing intentional forgetting again, it can be derived whether and what kind of memory-support material fosters the forgetting processes.

Analyses on team-level are important as a control variable for the individual performance. Since the team-members rely on each other, the individual learning curve depends on the overall team performance. E.g. when one worker in the chain is slow, both of the others will not work on that many products and thus practice less compared to a team where all members work quick and thus practice on a few more work pieces.

\section{Conclusion}

When new routines are about to be established, learning is only one important part of the process, with the process of forgetting as the other. Cues can facilitate and impede the retrieval of memory items, which facilitates or impedes forgetting. In theory it would be best for the acquisition of a changed working process to rebuilt the whole working space so nothing of the old can impede the encoding and recall of the new. Since this is highly impractical, this research tries to understand what can be done alternatively. Our experiments make first steps to measure intentional and cue-dependent forgetting, in order to answer questions like: Which changes and adaptations facilitate or hinder the establishment of new working routines? What should in practice be focused on when fundamental changes in the work environment are introduced?

\section{Acknowledgement}

The project is funded from 2016-2019 by the Deutsche Forschungsgemeinschaft (DFG), project numbers KL2207/6-1 and GR 1846/211.7.

\section{References}

[1] E. W. Tsang and S. A. Zahra, "Organizational unlearning," Human Relations, vol. 61, no. 10, pp. 1435-1462, 2008.

[2] P. M. de Holan and N. Phillips, "Remembrance of Things Past? The Dynamics of Organizational Forgetting," Management Science, vol. 50, no. 11, pp. 1603-1613, 2004.

[3] I. Nonaka and H. Takeuchi, The knowledge creation company: how Japanese companies create the dynamics of innovation. New York: Oxford University Press, 1995.

[4] H. Tsoukas and E. Vladimirou, "What is organizational knowledge?," Journal of management studies, vol. 38, no. 7, pp. 973-993, 2001.

[5] J. P. Walsh and G. R. Ungson, "Organizational memory," Academy of management review, vol. 16, no. 1, pp. 57-91, 1991.

[6] B. Hedberg, "How organizations learn and unlearn," Handbook of Organizational Design (1), pp. 3-27, 1981.

[7] A. J. Casey and F. Olivera, "Reflections on Organizational Memory and Forgetting," Journal of 
Management Inquiry, vol. 20, no. 3, pp. 305-310, Sep. 2011.

[8] A. E. Akgün, H. Keskin, J. C. Byrne, and G. S. Lynn, "Organizational unlearning as changes in beliefs and routines in organizations," Journal of OrgChange Mgmt, vol. 20, no. 6, pp. 794-812, Oct. 2007.

[9] A. E. Akgün, G. S. Lynn, and J. C. Byrne, "Antecedents and Consequences of Unlearning in New Product Development Teams," Journal of Product Innovation Management, vol. 23, no. 1, pp. 73-88, Jan. 2006

[10] M. S. Feldman, B. T. Pentland, L. D'Adderio, and N. Lazaric, "Beyond Routines as Things: Introduction to the Special Issue on Routine Dynamics," Organization Science, vol. 27, no. 3, pp. 505-513, May 2016.

[11] M. C. Becker, "Organizational routines: a review of the literature," Industrial and corporate change, vol. 13, no. 4, pp. 643-678, 2004.

[12] M. M. Crossan, H. W. Lane, and R. E. White, "An Organizational Learning Framework: From Intuition to Institution," The Academy of Management Review, vol. 24, no. 3, pp. 522-537, 1999.

[13] S. B. Klein, T. E. Robertson, and A. W. Delton, "Facing the future: Memory as an evolved system for planning future acts," Memory \& Cognition, vol. 38, no. 1, pp. 13-22, 2010.

[14] E. L. Bjork and R. A. Bjork, "Intentional forgetting can increase, not decrease, residual influences of to-be-forgotten information.," Journal of Experimental Psychology: Learning, Memory, and Cognition, vol. 29, no. 4, pp. 524-531, 2003.

[15] G. Mongillo, “G. Mongillo, O. Barak, and M. Tsodyks, Science 319, 1543 (2008).," Science, vol. 319, p. 1543, 2008.

[16] R. G. Crowder, "The Interference Theory of Forgetting in Long-Term Memory," Principles of Learning and Memory, 20-Nov-2014. [Online]. Available: https: / / www.taylorfrancis.com/. [Accessed: 29-Aug-2018]

[17] J. S. Nairne and J. N. Pandeirada, "Adaptive memory: Remembering with a stone-age brain," Current Directions in Psychological Science, vol. 17, no. 4, pp. 239-243, 2008.

[18] H. L. Roediger, Y. Weinstein, and P. K. Agarwal, "Forgetting: preliminary considerations," in Forgetting, Psychology Press, 2010, pp. 15-36.

[19] E. Tulving, “Cue-dependent forgetting: When we forget something we once knew, it does not necessarily mean that the memory trace has been lost; it may only be inaccessible," American Scientist, vol. 62, no. 1, pp. 74-82, 1974.

[20] B. Weiner, "Motivated forgetting and the study of repression," Journal of Personality, vol. 36, no. 2, pp. 213-234, 1968.

[21] A. Kluge and N. Gronau, "Intentional Forgetting in Organizations: The Importance of Eliminating Retrieval Cues for Implementing New Routines," Front. Psychol., vol. 9, 2018.
[22] D. Hislop, S. Bosley, C. R. Coombs, and J. Holland, "The process of individual unlearning: A neglected topic in an under-researched field," $\mathrm{Ma}$ nagement Learning, vol. 45, no. 5, pp. 540-560, Nov. 2014.

[23] T. Grisold, A. Kaiser, and J. Hafner, "Unlearning before Creating new Knowledge: A Cognitive Process.," 2017.

[24] A. Klammer and S. Gueldenberg, "Organizational Unlearning and Forgetting - a Systematic Literature Review," p. 16.

[25] I. H. Buchen, "Creating the future: innovation and the unlearning organization," Foresight, vol. 1, no. 2, pp. 117-123, Apr. 1999.

[26] R. A. Bjork, "Positive forgetting: The noninterference of Items intentionally forgotten," Journal of Verbal Learning and Verbal Behavior, vol. 9, no. 3, pp. 255-268, Jun. 1970.

[27] A. Baddeley, Essentials of Human Memory (Classic Edition). Psychology Press, 2013.

[28] V. Popov, I. Marevic, J. Rummel, and L. Reder, "Forgetting is a Feature, not a Bug: Intentionally Forgetting Some Things Helps Us Remember Others by Freeing up Working Memory Resources."

[29] S. Lass, H. Theuer, and N. Gronau, "A new approach for simulation and modeling of autonomous production processes," in System Science (HICSS), 2012 45th Hawaii International Conference on, 2012, pp. 1247-1256.

[30] K. Andresen, N. Gronau, and S. Schmid, "Ableitung von IT-Strategien durch Bestimmung der notwendigen Wandlungsfähigkeit von Informations systemarchitekturen," in Wirtschaftsinformatik 2005, O. K. Ferstl, E. J. Sinz, S. Eckert, and T. Isselhorst, Eds. Heidelberg: Physica-Verlag HD, 2005, pp. 63-82.

[31] S. Lass and N. Gronau, "Efficient Analysis of Production Processes with a Hybrid Simulation Environment," in Proceeding of the 22nd International Conference of Flexible Automation and Intelligent Manufacturing (FAIM 2012), Helsinki, Finnland, 2012.

[32] S. Lass, "Simulationskonzept zur Nutzenvalidierung cyber-physischer Systeme in komplexen Fabrikumgebungen," 2017.

[33] C. Dede, "Immersive interfaces for engagement and learning," science, vol. 323, no. 5910, pp. 66-69, 2009.

[34] M. Jerusalem and R. Schwarzer, "Skala zur allgemeinen Selbstwirksamkeitserwartung," Skalen zur Erfassung von Lehrer-und Schülermerkmalen. Dokumentation der psychometrischen Verfahren im Rahmen der Wissenschaftlichen Begleitung des Modellversuchs Selbstwirksame Schulen. Berlin: Freie Universität Berlin, 1999.

[35] B. Frank, Presence messen in laborbasierter Forschung mit Mikrowelten: Entwicklung und erste Validierung eines Fragebogens zur Messung von Presence. Springer-Verlag, 2014.

[36] B. Frank and A. Kluge, "Development and first validation of the PLBMR for lab-based microworld 
research," Proceedings of the International Society for Presence Research, pp. 17-19, 2014.

[37] A. Kluge, Wissenserwerb für das Steuern komplexer Systeme. Pabst Science Publ., 2004.

[38]M. Kersting, K. Althoff, and A. O. Jäger, WildeIntelligenz-Test 2: WIT-2. Hogrefe, Verlag für Psychologie, 2008.

[39] A. O. Jäger, H.-M. Süls s, and A. Beauducel, Berliner Intelligenzstruktur-Test: BIS-Test. Hogrefe, 1997.

[40] N. J. Cooke, J. C. Gorman, C. W. Myers, and J. L. Duran, "Interactive team cognition," Cognitive science, vol. 37, no. 2, pp. 255-285, 2013.

[41] R. D. Meyer, R. S. Dalal, and R. Hermida, "A review and synthesis of situational strength in the organizational sciences," Journal of Management, vol. 36, no. 1, pp. 121-140, 2010.

[42] B. T. Pentland and T. H\a erem, "Organizational routines as patterns of action: Implications for organizational behavior," Annu. Rev. Organ. Psychol. Organ. Behav., vol. 2, no. 1, pp. 465-487, 2015.

[43] T. Betsch, B. J. Brinkmann, K. Fiedler, and K. Breining, "When prior knowledge overrules new evidence: Adaptive use of decision strategies and the role of behavioral routines.," Swiss Journal of Psychology/Schweizerische Zeitschrift für Psychologie/Revue Suisse de Psychologie, vol. 58, no. 3, p. 151, 1999.

[44] J. W. Newstrom, "The Management of Unlearning: Exploding the" Clean Slate" Fallacy.," Training and Development Journal, vol. 37, no. 8, pp. 36-39, 1983.

[45] M. D. Cohen and P. Bacdayan, "Organizational routines are stored as procedural memory: Evidence from a laboratory study," Organization science, vol. 5, no. 4, pp. 554-568, 1994. 